\title{
Pain during Mammography: Impact of Breast Pathologies and Demographic Factors
}

\author{
R. Gupta ${ }^{a}$ M. Nayak ${ }^{b} \quad$ M. Khoursheed ${ }^{c}$ S. Roy ${ }^{a} \quad$ A.I. Behbehanic \\ Departments of aRadiology, ${ }^{\mathrm{b}}$ Psychiatry, and cSurgery, Faculty of Medicine, Kuwait University, Kuwait
}

Key Words

Pain · Mammography $\cdot$ Breast pathologies

\begin{abstract}
Objectives: The aim of this study was to systematically assess the pain experienced by patients undergoing mammography for various clinical presentations. Subjects and Methods: Two hundred and twenty-five patients aged $25-85$ years $(45.43 \pm 8.25$ years) presenting for mammography were included in the study. Presenting symptoms and clinical diagnosis were provided by the referring physicians and demographic information was obtained from self-reported questionnaires. Mammography results were recorded by the radiologist. Two different but reliable and valid measures of pain - Visual Analog Scale (VAS) and Pain/Discomfort Rating Scale (DRS) - were used to assess pain during mammography and data were statistically analyzed to examine the possible predictors of pain. Results: Forty-nine percent of the patients reported pain during mammography when cut-off level of VAS score was 40; however, when the cutoff level was raised to 60 (considering the preexisting pain as presenting symptom in some patients) only $23 \%$ reported pain. With DRS, $7 \%$ reported pain, $27 \%$ discomfort and $66 \%$ neither pain nor discomfort. Biserial corre-
\end{abstract}

lation between the VAS and DRS scores suggested strong positive agreement between the two measures of pain $(r=0.56$, d.f. $=90, p<0.01)$. Patients presenting with coexisting breast lumps and preexisting breast pain and those diagnosed with inflammatory conditions of the breast and fibrocystic changes experienced more pain during mammography. Conclusion: Preexisting breast pathologies and demographic factors such as age and educational level of the patient were important in reporting pain during mammography. This finding indicates that proper assessment of pain using standard measures and its association with breast pathologies and demographic factors is important for planning pain management in women undergoing mammography.

Copyright $\odot 2003$ S. Karger AG, Basel

\section{Introduction}

Mammography has been shown to be the most effective imaging technique available for the early diagnosis of breast cancer [1]. A certain amount of compression is needed during mammography in order to improve image quality and reduce absorbed radiation dose to the breast [2]. The compression is achieved by use of a plastic paddle, which presses the breast against the film cassette. The

\section{KARGER \\ Fax + 41613061234 \\ E-Mail karger@karger.ch \\ www.karger.com

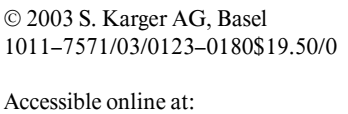

Dr. Renu Gupta

Associate Professor, Department of Radiology

Faculty of Medicine, Kuwait University

PO Box No. 24923, Safat 13110 (Kuwait)

Tel./Fax +965 53304 73, E-Mail renu@hsc.kuniv.edu.kw 
compression causes pain in some women and the reported incidence of pain varies from 0.2 to $62 \%[3,4]$. Hence the same degree of compression may cause little or no pain to some women and severe pain to others to the point of deterring them from wanting to undergo mammography [5-7]. Most of these studies were performed on women undergoing screening mammography $[3,4,6,8-10]$. The present study was undertaken to evaluate the incidence and severity of pain among symptomatic women undergoing mammography and to explore any possible association of pain during mammography with presenting symptoms, clinical and radiological diagnosis.

\section{Subjects and Methods}

This study was approved by the Ethics Committee, Faculty of Medicine, Kuwait University, Kuwait. Informed consent was obtained from all the subjects. Two hundred and twenty-five women referred for mammography by their physicians or surgeons were recruited for the study. Exclusion criteria were mental retardation, disorientation and serious physical illness as these could affect the ability to understand and complete the questionnaire. The subjects ranged in age from 25 to 85 years $(45.43 \pm 8.25$ years). The majority of them were referred for mammography due to breast-related symptoms of pain 81 , lump 74 , or discharge 25 . One hundred and sixty patients were referred for evaluation of one reason only, 61 for 2 reasons, 5 for 3 reasons or more. Forty-five patients were referred for pre-hormone replacement therapy and 16 for family history of breast cancer (table 1).

Compression was achieved by use of a plastic paddle which pressed the breasts against the film cassette. Compression was considered sufficient when the skin of the breast became taut. Mammography was conducted by female radiographers who have experience of performing approximately 1,300-1,500 of mammograms annually. Mammograms were performed on Senograph DMR mammographic unit. Two standard views (mediolateral oblique and craniocaudal) of each breast were routinely obtained. Additional views were taken if necessary.

Patients were asked to complete a brief questionnaire regarding pain information shortly after the mammogram. Radiologist reported the mammograms. Two previously validated pain measures were used, the Visual Analog Scale (VAS) and Pain/Discomfort Rating Scale (DRS) $[4,9,11,12]$. The VAS measure used in this study comprised a $10-\mathrm{cm}$ line whose ends were labeled 'No pain' and 'Worst pain', respectively. Subjects were required to mark the line at a spot corresponding to the intensity of pain they experienced. The DRS was used as a second measure and subjects were asked to choose among the following categorical descriptions: very comfortable, mildly comfortable, uncomfortable but tolerable, very uncomfortable, painful/very painful to describe accurately their experience during mammography.

All subjects provided a score on the VAS as a measure of the intensity of pain experienced and a descriptive categorization of the pain on the DRS scale.
Table 1. Clinical presentations

\begin{tabular}{llc}
\hline Presentation & $\mathrm{n}$ & Percentage \\
\hline Pain alone & 81 & 36 \\
Lump alone & 74 & 33 \\
Discharge & 25 & 11 \\
Lump and pain & 14 & 6 \\
Pre HRT & 45 & 20 \\
Family history & 16 & 7
\end{tabular}

Note: Some of the patients had more than one symptom. HRT $=$ Hormone replacement therapy.

\section{Demographic Information}

Age, educational level and nationality were obtained from a selfreport questionnaire. The referring doctor provided findings on clinical examination and clinical diagnosis.

\section{Data Analysis}

Data were statistically analyzed using the SPSS software package version 11.0 and $p<0.05$ was considered as significant. A similar series of comparative analyses using Student's t tests, or one-way analyses of variance (ANOVAs) were conducted to assess the influence of demographic and medical variables on VAS scores. To facilitate data analyses the DRS scores were collapsed into 3 comprehensive categories: pain, discomfort and neither pain nor discomfort.

\section{Results}

The mammographic findings were as follows: normal, 128 (57\%); benign conditions such as fibroadenoma, 25 (11\%); fibrocystic changes, $40(18 \%)$; inflammatory diseases and ductal ectasia, 10 (4\%); malignant lesions, 17 $(8 \%)$; suspicious for malignancy, 3 (1\%), and miscellaneous, 2 patients.

Numerical and descriptive ratings of pain experienced by the women during mammography are given in table 2 . Most of the women (96\%) reported some degree of pain. With a VAS cut-off level of 40, $110(49 \%)$ of the women reported pain. However, when the cut-off level was raised to 60 to take into account pre-existing pain, only $52(23 \%)$ reported pain during mammography. Women referred with both breast lumps and pre-existing pain, as a group had a significantly higher average $(p<0.05)$ VAS score (45.71) than women with either breast lumps or pre-existing pain (37.46) and women with neither breast lump nor breast pain $(37.47)(p<0.05)$. Age and educational levels were important factors in reporting pain. Thirty-two per- 
Table 2. Pain descriptive and numerical ratings of subjects undergoing mammography

\begin{tabular}{lcr}
\hline Score or score range & \multicolumn{2}{l}{ Cases } \\
\cline { 2 - 3 } & $\mathrm{n}$ & $\%$ \\
\hline \multicolumn{2}{l}{ Visual analog scale score (intensity of pain) } \\
0 & 10 & 4 \\
$5-15$ & 26 & 12 \\
$20-30$ & 79 & 35 \\
$40-50$ & 59 & 26 \\
$60-70$ & 30 & 13 \\
$80-90$ & 20 & 9 \\
100 & 1 & 1 \\
Total & 225 & 100
\end{tabular}

Discomfort Rating Scale (descriptive category)

Very comfortable

Comfortable

\begin{tabular}{rr}
21 & 9 \\
92 & 41 \\
35 & 16 \\
55 & 24 \\
6 & 3 \\
16 & 7 \\
\hline 225 & 100 \\
\hline
\end{tabular}

Mildly comfortable

Uncomfortable but tolerable

Very uncomfortable

Painful/very painful

Total

Table 3. VAS scores based on educational levels

\begin{tabular}{ll}
\hline Educational level & Mean score \\
\hline None & 38.60 \\
Primary & 32.69 \\
Intermediate & 31.03 \\
High school & 36.85 \\
College education & 40.22 \\
Graduate & 42.69 \\
Post-graduate & 45.21 \\
\hline
\end{tabular}

cent of patients below the age of 40 years reported pain as compared to $21 \%$ of those more than 40 years old. Women with postgraduate level education had the highest VAS score of 45.21 (table 3 ) and the difference was statistically significant $(\mathrm{p}<0.05)$.

The DRS scores showed that $16(7 \%)$ reported pain, 61 (27\%) discomfort and 148 (66\%) subjects neither pain nor discomfort. Analyses using the 3 collapsed categories of DRS (pain, discomfort, neither discomfort nor pain) showed no differences by nationality, reason for clinical referral, breast density or mammographic diagnosis as
$28 \%$ of women with clinical diagnosis of fibrocystic disease, $24 \%$ with fibroadenoma, $24 \%$ with diagnosis of malignancy and $22 \%$ with no abnormality reported pain during mammography. However, $39 \%$ of women with both lumps and pre-existing pain, $21 \%$ of women with either breast lump or pre-existing pain and $27 \%$ of women with neither lump nor pre-existing pain reported pain. Biserial correlation between the VAS and DRS scores suggested a strong positive agreement between the two measures of pain $(r=0.56$, d.f. $=90, p<0.01)$.

\section{Discussion}

This study on pain associated with mammography demonstrates the use of standardized measures of pain such as VAS and DRS. The VAS scoring system is a useful, valid and easy tool for assessment of pain $[11,12]$. It enables patients with varying levels of education to provide an indication of the intensity of pain/discomfort (table 3).

A significant association was found between educational level and discomfort rating, similar to a previous report [4]. This finding supports the notion that women with college level education are better able to verbally categorize their experience and hence be better able to use a scale such as the DRS that uses categorical response options. Interestingly women without education had a higher VAS score than women with limited education. A plausible explanation is that the radiographer carefully explained the scoring system to the women without education while it was assumed that those with limited education could read the VAS scoring system themselves. This finding points out the need for providing adequate explanation to both groups since English is not their primary language.

The present study extends research on pain and discomfort during mammography by including symptomatic women, unlike previous studies that included mostly women undergoing screening mammograms $[3,4,6,8-$ $10]$. Consistent with previous studies $[4,10]$ extreme pain was rarely reported in our study as only $23 \%$ of the patients found mammography to be painful. As would be expected, patients with inflammatory conditions of breast and fibrocystic disease most often reported pain during mammography. Presenting symptoms most often associated with mammographic pain were coexistence of breast lump and pre-existing breast pain.

It is worthy to point out that this study included women of a wide age range (25-85 years) unlike previous stud- 
ies done on women over 50 years of age $[4,8,10,13]$, as $32 \%$ of women below 40 years of age reported pain during mammography indicating a need to pay attention to this age group that is not normally screened for mammography. Systematic assessment of pain using standard measures provides information on intensity of pain and is imperative for planning pain management in women undergoing mammography. Findings of previous studies indicate an association between pain during mammography and behaviour of staff on one hand, and the useful role of behavioral techniques, exemplified by systematic relaxation and psychological interventions such as distraction in reducing pain on the other hand [10, 14-17]. Given the occurrence of pain during mammography as observed in this and other studies $[4,8,10,13]$ and reports indicating that experience of pain might deter some women from undergoing future mammography [6, 15], we recommend the use of aforementioned behavioral techniques to distract pain for women undergoing mammography particularly those presenting with symptomatic breasts.

\section{Conclusion}

The present study has shown that $23 \%$ of the patients experienced pain during mammography. Women with fibrocystic changes and inflammatory conditions and those presenting with both breast lump and pre-existing pain are more likely to experience pain. Age and educational level were also important factors in reporting pain during mammography. Further proper assessment of pain using validated measures is important for planning pain management in patients such as those undergoing mammography.

\section{Acknowledgement}

This project was funded by The Research Management Unit, Kuwait University project No. MDT 304. I would like to thank Mrs. Delphine D'Souza for typing the manuscript.

\section{References}

1 Drukker BH: Breast disease: A primer on diagnosis and management. Int $\mathrm{J}$ Fertil Women Med 1997;42:278-287.

2 Poulos A, Richard M: Compression in mammography and the perception of discomfort. Australas Radiol 1997;41:247-252.

3 Keefe FJ, Hauck ER, Egert J, Rimer B, Kornguth P: Mammography pain and discomfort: A cognitive-behavioral perspective. Pain 1994; 56:247-260.

4 Kornguth PJ, Keefe FJ, Conway MR: Pain during mammography: Characteristics and relationship to demographic and medical variables. Pain 1996;66:187-194.

5 Andrews FJ: Pain during mammography: Implications for breast screening programmes. Australas Radiol 2001;45:113-117.

6 Keemers-Gels ME, Groenendijk RP, Van den Heuvel JH, Boetes C, Peer PG, Wobbes TH: Pain experienced by women attending breast cancer screening. Breast Cancer Res Treatm 2000;60:235-240.
7 Chartres S, Doyle T, Elwood M, Nicoll J, Rowe K, Swann M, Watt A: Is mammography such a pain? NZ Med J 1998;111:371.

8 Aro Ar, Absetz-Ylostalo P, Eerola T, Pamilo $\mathrm{M}$, Lonnquist $\mathrm{J}$ : Pain and discomfort during mammography. Eur J Cancer 1996;32A:16741679.

9 Stomper PC, Kopan DB, Sandwsky N, Sonenfeld MR, Swann CA, Gelman RS, Meyer JE, Jochelson MS, Hunt MS, Allen PD: Is mammography painful? A multicenter patient survey. Arch Intern Med 1988;148:521-524.

10 Kashikar-Zuck S, Keefe FJ, Kornguth P, Beaupre P, Holzberg A, Delong D: Pain coping and the pain experience during mammography: A preliminary study. Pain 1997;73:165172.

11 Gramling SE, Elliot TR: Efficient pain assessment in clinical settings. Behav Res Ther 1992; 30:71-73.
12 Harrison A: Comparing nurse's and patient's pain evaluations: A study of hospitalized patients in Kuwait. Soc Sci Med 1993;36:683692.

13 Dullum JR, Lewis EC, Mayor JA: Rates and correlates of discomfort associated with mammography. Radiology 2000;214:547-552.

14 Bryla CM: The relationship between stress and the development of breast cancer: A literature review. Oncol Nurses Forum 1996;23:441448.

15 Bruyninckx E, Mortelmans D, Van Goethem M, Van Hove E: Risk factors of pain in mammographic screening. Soc Sci Med 1999;49: 933-941.

16 Gupta R, Nayak MB, Khourseed M, Roy S: Emotional distress in women presenting for breast imaging. Ann Saudi Med 1999;19:511514.

17 Vaile MSB, Calnan M, Rutter DR, Wall B: Breast cancer screening services in three areas: Uptake and satisfaction. J Publ Hlth Med 1993; 15:37-45. 